\title{
Improving a Power Line Communications Standard with LDPC Codes
}

\author{
Christine Hsu, ${ }^{1}$ Neng Wang, ${ }^{1,2}$ Wai-Yip Chan, ${ }^{1}$ and Praveen Jain ${ }^{1}$ \\ ${ }^{1}$ Department of Electrical and Computer Engineering, Queen's University, Kingston, ON, Canada K7L 3N6 \\ ${ }^{2}$ Nortel Networks, Richardson, TX 75082-4399, USA
}

Received 31 October 2006; Revised 7 March 2007; Accepted 4 May 2007

Recommended by Lutz Lampe

\begin{abstract}
We investigate a power line communications (PLC) scheme that could be used to enhance the HomePlug 1.0 standard, specifically its ROBO mode which provides modest throughput for the worst case PLC channel. The scheme is based on using a low-density parity-check (LDPC) code, in lieu of the concatenated Reed-Solomon and convolutional codes in ROBO mode. The PLC channel is modeled with multipath fading and Middleton's class A noise. Clipping is introduced to mitigate the effect of impulsive noise. A simple and effective method is devised to estimate the variance of the clipped noise for LDPC decoding. Simulation results show that the proposed scheme outperforms the HomePlug 1.0 ROBO mode and has lower computational complexity. The proposed scheme also dispenses with the repetition of information bits in ROBO mode to gain time diversity, resulting in 4 -fold increase in physical layer throughput.
\end{abstract}

Copyright (c) 2007 Christine Hsu et al. This is an open access article distributed under the Creative Commons Attribution License, which permits unrestricted use, distribution, and reproduction in any medium, provided the original work is properly cited.

\section{INTRODUCTION}

Power line communications (PLC) have received increasing attention due to the wide availability of power lines, even though PLC face the challenge of harsh and noisy transmission channels. HomePlug 1.0 is a current industry standard for PLC in North America [1]. It uses concatenated Reed-Solomon (RS) and convolutional forward error correction (FEC) with interleaving for coding payload data and orthogonal frequency division multiplexing (OFDM) for modulation. HomePlug guarantees rates from 1-14 Mbps for the physical layer (PHY) throughput. The low-end $1 \mathrm{Mbps}$ throughput is obtained when operating the robust-OFDM (ROBO) mode over severely degraded channels. The goal of this paper is to improve the low-end throughput. As we describe below, the throughput can be increased considerably with no increase in complexity.

The convolutional decoder in ROBO mode performs hard decision decoding. Recently, various studies have been carried out to investigate the application of various powerful FEC techniques with soft decision decoding, such as turbo codes and low-density parity-check (LDPC) codes, to PLC and reported promising results [2-4]. Ardakani et al. [2] model the PLC channel by the concatenation of an additive white Gaussian noise (AWGN) channel with an erasure channel. Umehara et al. [3] and Nakagawa et al. [4] use the Middleton class A noise (AWCN) model [5] to simulate the impulsive noise for the PLC channel. The Middleton class A noise model has been shown in the literature to be valid for impulsive noise in PLC (see, e.g, Häring and Vinck [6]). However, most of the existing studies involving AWCN focus on investigating single carrier cases and/or do not consider the effect of multipath fading. Multipath fading occurs when a propagating signal is corrupted by reflections caused by impedance mismatches. Ma et al. [7] examine the effects of impulsive noise and multipath fading on OFDM for uncoded signals. Most of these investigations are compartmentalized by looking at only selected aspects of PLC schemes.

Towards our aim of improving the HomePlug standard ROBO mode, we take a more comprehensive approach by studying the overall performance of OFDM with LDPC codes over PLC channels modeled by both AWCN and multipath fading. We first assess the performance of OFDM over the AWCN channel. It is observed that the time-frequency transformation of OFDM spreads the AWCN across subcarriers, effectively transforming the AWCN into additive white Gaussian noise (AWGN). In channels with severe impulsive noise, however, noise clipping is used to improve error rate performance. A simple and efficient method is devised to estimate the variance of the clipped noise for LDPC decoding. 


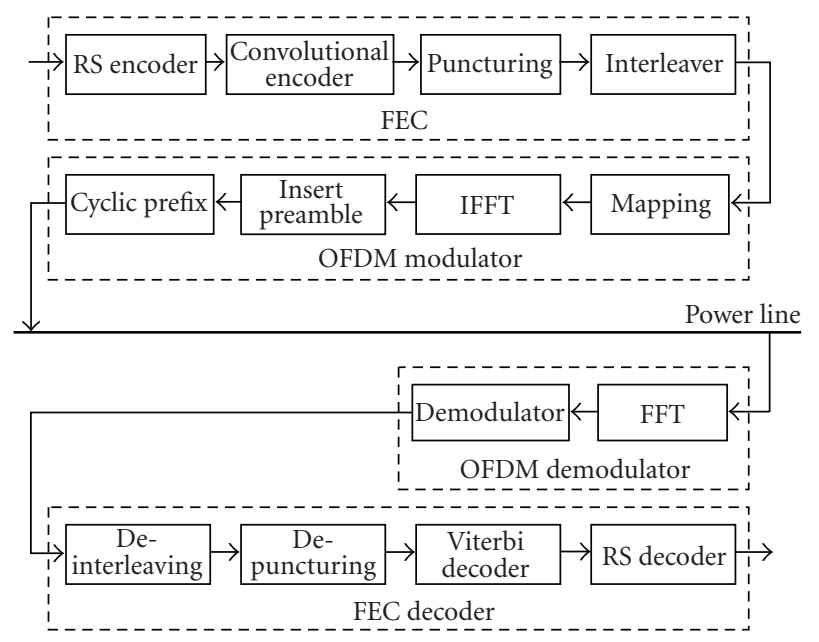

Figure 1: The HomePlug 1.0 Standard [1].

The method works remarkably well for highly impulsive noises. We compare the performance of our proposed approach to that of HomePlug 1.0 by computer simulation. The results show that our proposed approach outperforms the HomePlug 1.0 ROBO mode, while the computational complexity per decoded bit is reduced. Moreover, our approach does not involve the repeated transmission of information that is done in ROBO mode to gain time diversity; thus, information throughput in the physical layer is increased.

In Section 2, we provide a brief background on the HomePlug 1.0 standard, LDPC codes, and AWCN. Section 3 examines the performance of various LDPC codes over an AWCN channel. In Section 4, the performance of OFDM over the AWCN channel is analyzed, a noise clipping rule is described and a simple theoretical approximation of the post-OFDM variance of the clipped noise is proposed. Section 5 compares the simulation results of our proposed approach and the HomePlug 1.0 ROBO mode, and discusses issues of complexity, implementation and efficiency.

\section{BACKGROUND}

In this section, we provide a brief description of the HomePlug 1.0 standard, the encoding and decoding procedures for LDPC codes, as well as the AWCN model for impulsive noise.

\subsection{HomePlug 1.0 standard}

In HomePlug 1.0, the PHY layer employs OFDM transmission. An overall block diagram of the HomePlug 1.0 standard is given in Figure 1. OFDM transmits information over a number of subcarriers in parallel. It has the benefit of robustness to multipath and low-complexity equalization. In cases where the channel is severely degraded, or where channel estimation has not been performed, the ROBO mode is used. All 84 carriers are used for this mode. Differential binary phase shift keying (DBPSK) modulation and concatenated RS and convolutional coding with interleaving are applied. The RS code-rate ranges from $31 / 39$ to $43 / 51$ and supports transmission blocks of 40 OFDM symbols. A block interleaver is employed with the number of columns equal to half the number of OFDM symbols. Convolutionally, encoded data is written by rows and read by columns with a shift in starting row for each successive column read. The interleaver is read four times to provide a copy code, with each copy shifted in frequency by $21(=84 / 4)$ OFDM carriers. This extensive time and frequency diversity enables robust operation under hostile channel conditions. The bit repetition introduces extra redundancy that reduces the data rate to $1 / 4$ bit per carrier per OFDM symbol for the ROBO modulation.

\subsection{LDPC codes}

LDPC codes were originally introduced by Gallager in 1963 [8]. Gallager defines LDPC codes as those specified by a sparse parity-check matrix $\mathbf{H}$ containing mostly 0 's and relatively few 1's, satisfying

$$
x \cdot \mathbf{H}^{T}=0,
$$

where $x=\left(x_{0}, x_{1}, x_{2}, \ldots, x_{L-1}\right)$ is the codeword vector. Each column of $\mathbf{H}$ corresponds to a coded bit and each row of $\mathbf{H}$ represents a parity-check sum. The location of a " 1 " in the $(i, j)$ position of $\mathbf{H}$ indicates that the $j$ th coded bit is required for the $i$ th parity check sum. The sparse matrix structure is especially suitable for iterative decoding algorithms. It has been reported that, among various decoding methods for LDPC codes, the sum-product algorithm (SPA) based on probability propagation offers capacity-approaching performance close to the more complex turbo codes [9]. SPA performs iterative decoding through the passing of soft messages in terms of likelihood ratios between the coded bits and the check sums. The likelihood ratio is initialized as

$$
\mathrm{LR}=\frac{P\left(r_{n} \mid x_{n}=1\right)}{P\left(r_{n} \mid x_{n}=0\right)},
$$

where $r_{n}$ and $x_{n}$ denote received sample and transmitted bit, respectively. Equation (2) represents a channel reliability factor influenced by channel characteristics. SPA decoding allows implementation for high data rates using fully parallel processing, in which all code-bit messages or all check-sum messages are computed concurrently. The selection of a suitable LDPC code for PLC is addressed below in Section 3. In our simulation, an efficient MacKay's algorithm for LDPC decoding based on LR is adopted. In practice, a more numerically stable algorithm using log likelihood ratio (LLR) for LPDC decoding, such as [4], may be preferred.

\subsection{AWCN}

The received signal in single carrier systems is modeled as

$$
r_{n}=x_{n}+z_{n}
$$

where $x_{n}$ is the transmitted signal and $z_{n}$ is the channel noise. In the case of a normalized complex Middleton's class A 


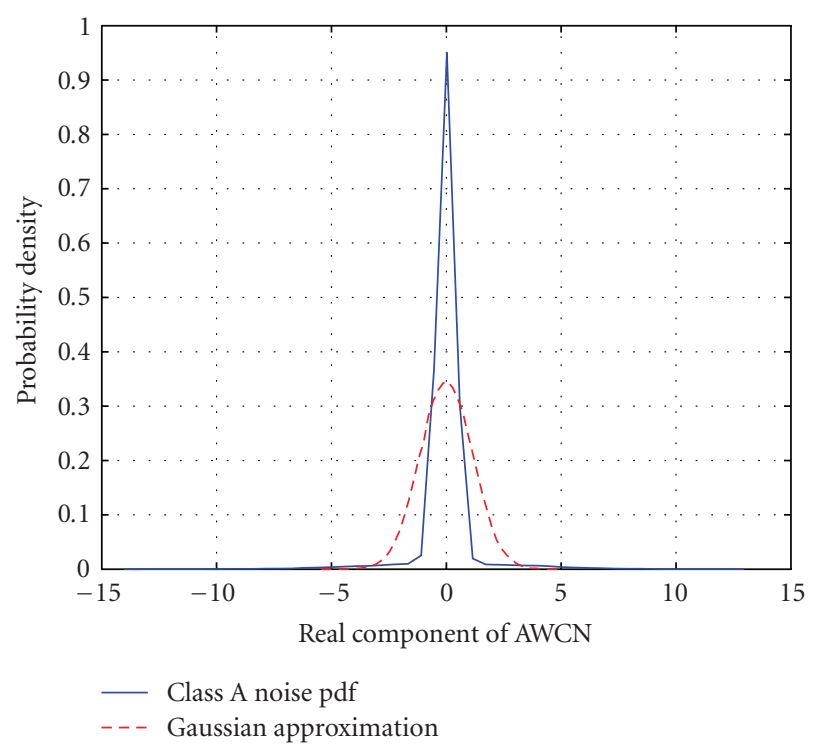

FIGURE 2: Probability density function of real component of AWCN $\left(A=0.1, \mathrm{GIR}=0.1, \sigma^{2}=1.3095\right)$.

noise, $z_{n}$ has the probability density function (pdf) given by [5]

$$
p_{z}\left(z_{n}\right)=\sum_{m=0}^{\infty} \frac{e^{-A} A^{m}}{m !} \cdot \frac{1}{2 \pi \sigma_{m}^{2}} \exp \left(-\frac{\left|z_{n}\right|^{2}}{2 \sigma_{m}^{2}}\right),
$$

with

$$
\sigma_{m}^{2}=\sigma^{2}\left(\frac{m / A+\Gamma}{1+\Gamma}\right)
$$

where $A$ is the impulsive index which measures the average number of impulses over the signal period, $\Gamma\left(=\sigma_{G}^{2} / \sigma_{I}^{2}\right)$ is the Gaussian-to-impulsive power ratio (GIR) with Gaussian noise variance $\sigma_{G}^{2}$, impulsive noise power (variance) $\sigma_{I}^{2}$, and total variance $\sigma^{2}=\sigma_{G}^{2}+\sigma_{I}^{2}$. The Gaussian variance is determined by

$$
\sigma_{G}^{2}=\frac{1}{2 R\left(E_{b} N_{0}\right)},
$$

where $R$ is the code rate and $E_{b} / N_{o}$ is the input signal-tonoise ratio (SNR). In our simulation, a third-order approximate pdf of a normalized complex AWCN is adopted (i.e., $m=0,1,2,3)$ as an adequate representation of the theoretical pdf. For highly impulsive channels $(A<1)$, the thirdorder approximate pdf accounts for more than $99 \%$ of the probability mass of the pdf in (4). Increasing $m$ beyond 3 has little impact on the results reported below. Equation (4) can then be approximated by

$$
p_{z}(z)=\sum_{m=0}^{3} \frac{e^{-A} A^{m}}{m !} \cdot \frac{1}{2 \pi \sigma_{m}^{2}} \exp \left(-\frac{|z|^{2}}{2 \sigma_{m}^{2}}\right) .
$$

Figure 2 shows the pdf of the real part of a typical AWCN. A Gaussian pdf with the same total variance $\sigma^{2}$ is also given. It can be seen that the AWCN pdf has a narrower and higher peak around zero and longer tails, as compared to the Gaussian pdf with the same variance.

For most of the simulations presented in this paper, $A$ and GIR are both set to 0.1 . These parameters correspond to a "heavily disturbed environment" from field measurements (see Ma et al. [7] and Zimmermann and Dostert [10]) and commensurate with our goal of improving the ROBO mode which is used in the hostile channel condition.

\section{SELECTION OF LDPC CODE}

To select an LDPC code for PLC, we compare the performance of different LDPC coding schemes. Six LDPC coding schemes are compared, namely, Euclidean geometry (EG) code [11], random regular code (MacKay) [12], array code [13], random irregular code (MacKay) [12], modified array code [14], and optimized irregular code [15]. To assess the robustness of these codes under severe impulsive noise conditions, the candidate codes with a low code rate $(\approx 0.51)$ and a moderate length (2000) are assessed for single carrier system over an AWCN channel with $A=0.1$ and GIR $=0.001$. The initial likelihood ratio (2) for SPA decoding of a BPSK signal, after incorporating (7), becomes

$$
\begin{aligned}
\operatorname{LR} & =\frac{P\left(r_{n} \mid x_{n}=+1\right)}{P\left(r_{n} \mid x_{n}=-1\right)} \\
& =\frac{\sum_{m=0}^{3}\left(e^{-A} A^{m} / m !\right) \cdot\left(1 / \sqrt{2 \pi} \sigma_{m}\right) \exp \left(-\left(r_{n}-1\right)^{2} / 2 \sigma_{m}^{2}\right)}{\sum_{m=0}^{3}\left(e^{-A} A^{m} / m !\right) \cdot\left(1 / \sqrt{2 \pi} \sigma_{m}\right) \exp \left(-\left(r_{n}+1\right)^{2} / 2 \sigma_{m}^{2}\right)},
\end{aligned}
$$

where $r_{n}$ is the real component of the received signal. The results for a single carrier system are presented in Figure 3, as bit error-rate (BER) versus signal-to-noise ratio (SNR) performance. (The background Gaussian noise power is used to calculate the SNR.)

It can be seen that the two random codes, regular and irregular MacKay codes, and the irregular optimized codes outperform others. This could be attributed to the more random-like structure of the random codes and the optimized code. Further, the random MacKay codes have the least numbers of short cycles (of length 4,6, and 8), which are detrimental to proper decoding of an LDPC code. Among these three codes, the regular MacKay code offers the best performance, while the irregular optimized code shows an error floor with increasing SNR. Consequently, most of the results in the rest of this paper are for employing the regular MacKay code.

\section{IMPULSIVE NOISE ANALYSIS}

\subsection{OFDM over AWCN}

PLC channels often provide multiple propagation paths to the transmitted signal. OFDM is well suited for ameliorating the frequency selectivity of multipath channels. However, as we demonstrate below, OFDM performance can be severely degraded by the presence of strongly impulsive noise. In this 


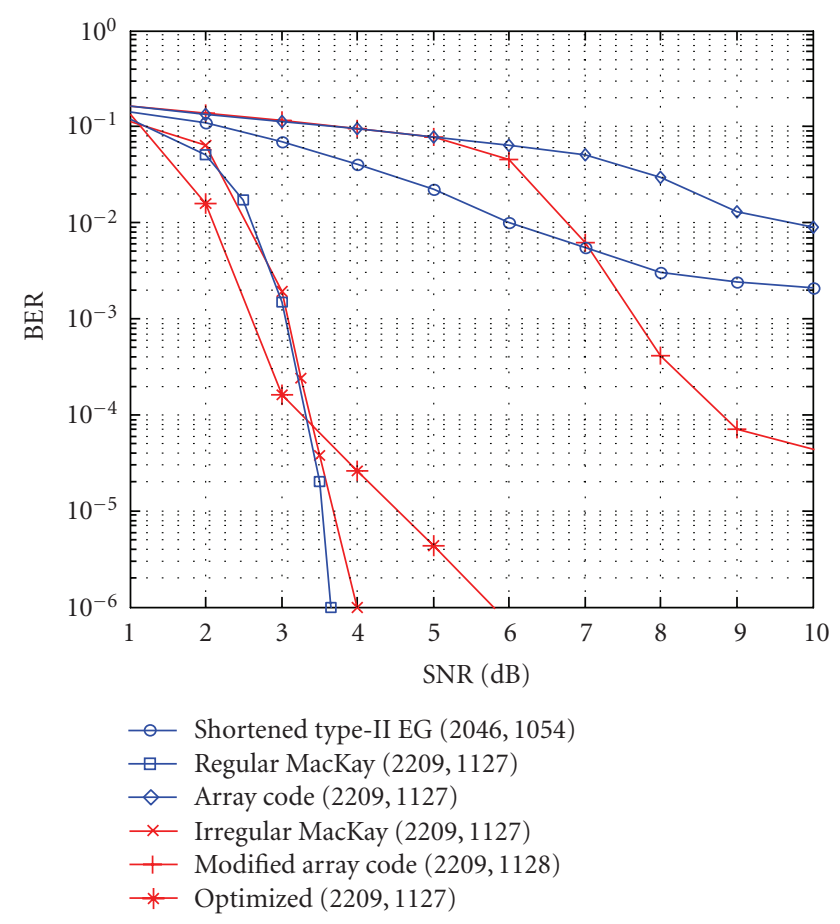

FIGURE 3: BER versus SNR for a single carrier system with LDPC codes (code rate $\approx 0.51)$ over an AWCN channel $(A=0.1$, GIR $=$ 0.001).

section, we consider clipping the received OFDM signal as a means to improve performance over AWCN channels. The results in this section are for OFDM over AWCN channels with no multipath fading.

The OFDM receiver's FFT operation sums and spreads impulses evenly over the range of frequencies. With a sufficiently large FFT length, the FFT-transformed noise approaches a Gaussian distribution [16]. It should be noted that, for N-point FFT, each frequency bin has noise due to time-domain impulsive noise, which is a summation of $\mathrm{N}$-phase-modulated version of the original time-domain AWCN. Therefore, in frequency-domain, it is still AWCN but not as impulsive as the original time-domain AWCN. Its behavior is between AWGN and AWCN. While we have chosen an encoder best suitable for AWCN, the above property allows us to approximate decoding for an effective AWGN to simplify the decoder. Simulation results justify this simplification. The pdf of the frequency-domain noise $Z$ can be approximated by a Gaussian distribution $\mathcal{N}\left(0, \sigma_{Z}^{2}\right)$ with variance

$$
\sigma_{Z}^{2}=\frac{1}{N} \sum_{n=0}^{N-1} \sigma_{z_{n}}^{2}=\sigma_{G}^{2}\left(1+\frac{1}{\Gamma}\right)
$$

where $N$ is the FFT length. The subsequent LDPC decoding then amounts simply to a straightforward LDPC-AWGN decoding. The channel likelihood ratio can be obtained as

$$
\mathrm{LR}=\exp \left(\frac{2 r_{n}}{\sigma_{Z}^{2}}\right)=\exp \left(\frac{2 r_{n}}{\sigma_{G}^{2}(1+1 / \Gamma)}\right)
$$

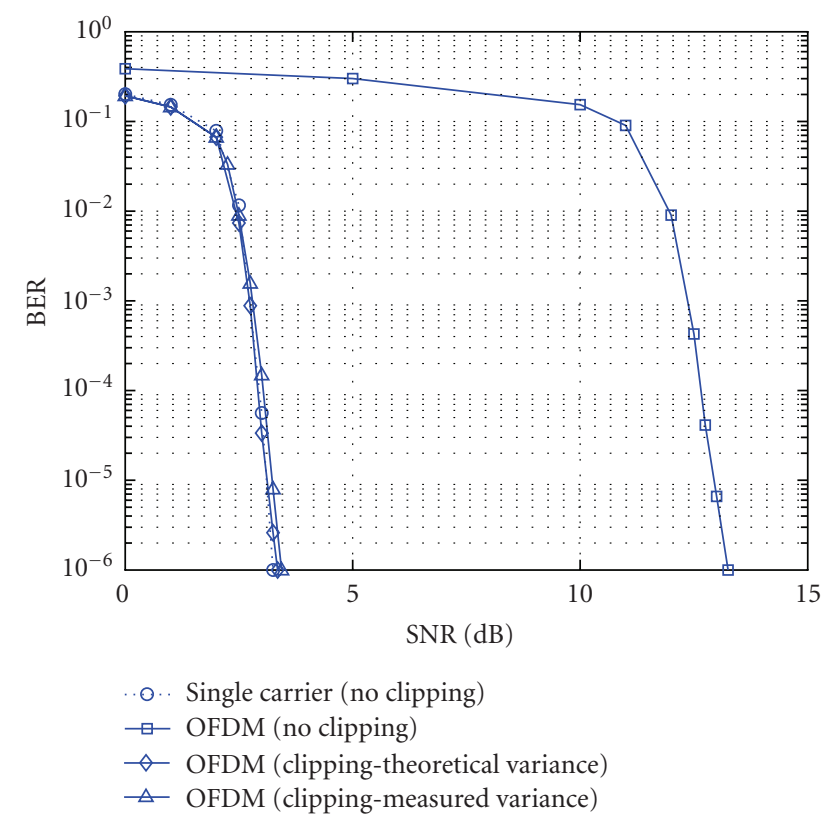

FIGURE 4: BER versus SNR for single carrier and OFDM systems with LDPC $(3312,1397)$ coding over AWCN channel $(A=0.1$, $\mathrm{GIR}=0.1)$.

According to our simulations, this works well for moderateto-high GIR. In channels with severe impulsive noise, however, the BER performance is not satisfactory as the frequency domain noise inherits the pre-FFT high noise variance in the time domain. This effect is illustrated by the BER versus SNR curve given in Figure 4. It can be seen that the conventional OFDM does not perform well over a noisy AWCN channel, compared to the single carrier case. In order to alleviate the effect of impulsive noise, the spreading effect of OFDM is advantageously exploited through time-domain noise clipping: the detection and elimination of possible impulsive noise can be performed in the time domain without suppressing the information bit in the frequency domain.

\subsection{OFDM over AWCN with clipping}

Various impulse noise mitigation techniques have been studied for uncoded OFDM systems. Notably, Häring and Vinck [17] proposes an efficient OFDM demodulation and detection scheme that can suppress the effect of impulsive noise. As we are dealing with an LDPC coded OFDM system, a simpler method based on impulse clipping is adopted from Suraweera et al. [18]:

$$
\tilde{r}_{n}= \begin{cases}r_{n} & \text { if }\left|r_{n}\right| \leq \mathrm{Amp}, \\ 0 & \text { if }\left|r_{n}\right|>\text { Amp }\end{cases}
$$

where Amp is a threshold amplitude set to some multiple of the standard deviation of the background Gaussian noise and is experimentally determined in this paper. 
Since AWCN noise can be considered as a mixture of Gaussian noises, the above clipping procedure will result in a mixture of doubly-truncated Gaussian noises with a normalized variance adjustment factor $V$ given in [19] as follows:

$$
V=1+\frac{x_{L} f\left(x_{L}\right)-x_{R} f\left(x_{R}\right)}{F\left(x_{R}\right)-F\left(x_{L}\right)}-\left[\frac{f\left(x_{L}\right)-f\left(x_{R}\right)}{F\left(x_{R}\right)-F\left(x_{L}\right)}\right]^{2},
$$

where $f(\cdot)$ and $F(\cdot)$ denote the pdf and cumulative distribution function (cdf) of the untruncated standard normal distribution $\mathcal{N}(0,1)$, and $x_{L}$ and $x_{R}$ the left and right truncating points, respectively.

The resulting noise variance of the clipped signals can be estimated as follows. Using Bussgang's theorem (see Dardari et al. [20]) which relates the input and output of a memoryless nonlinearity, the output of the clipper mapping in (11) can be expressed as

$$
\tilde{r}_{n}=k r_{n}+z_{c}=k x_{n}+k z_{n}+z_{c},
$$

where $k, 0<k<1$, is a constant "shrinking factor" and $z_{c}$ is the clipping noise. Readers are referred to $[18,20]$ for a discussion of clipping and shrinking factor. The net effect is that $z_{c}$ acts to null the large impulses but also distorts the signal. The effect on the resulting variance of the clipped signal can be implicitly estimated through a simple probability approach. The resulting variance has two components: a reduced noise variance due to clipping of the impulsive noise and an additional noise variance introduced by clipping the signal, denoted by $\Delta^{-}$and $\Delta^{+}$, respectively. Both $\Delta^{-}$ and $\Delta^{+}$can be approximated by a weighted sum of $\Delta_{m}^{-}$and $\Delta_{m}^{+}$from individual impulse noise components corresponding to $m=0,1,2,3$ as given in (7).

From (12) with symmetrical truncation, it can be shown that

$$
\begin{aligned}
\Delta_{m}^{-} & =V\left(2 \sigma_{m}^{2}\right) \\
& =\left[1+\frac{-a_{m} f\left(-a_{m}\right)-a_{m} f\left(a_{m}\right)}{F\left(a_{m}\right)-F\left(-a_{m}\right)}\right]\left(2 \sigma_{m}^{2}\right),
\end{aligned}
$$

where $a_{m}=\operatorname{Amp} /\left(\sqrt{2} \sigma_{m}\right)$. The variance introduced by clipping of the signals corresponds to lost power of the clipped signals, which is given by

$$
\Delta_{m}^{+}=2\left[1-F\left(a_{m}\right)\right]
$$

The total noise variance due to clipping can be obtained as

$$
\begin{aligned}
\tilde{\sigma}_{z}^{2} & =\Delta^{-}+\Delta^{+} \\
& =\sum_{m=0}^{3} \frac{e^{-A} A^{m}}{m !}\left(\Delta_{m}^{-}+\Delta_{m}^{+}\right) .
\end{aligned}
$$

The noise variance in the frequency domain is $\tilde{\sigma}_{Z}^{2}=\tilde{\sigma}_{z}^{2}$. The decoding can again be carried out simply as LDPC-AWGN decoding but with a significantly reduced noise variance as a result of impulse clipping in the time domain. From (13) and (14), it can be seen that the total variance of clipped noise depends on the choice of the clipping threshold Amp. There is

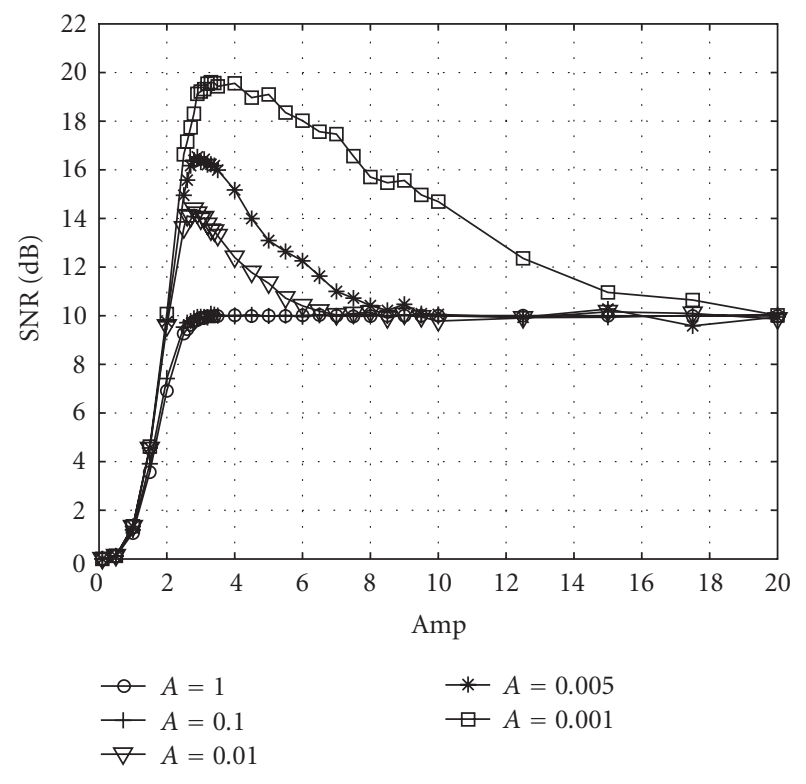

FIgURE 5: SNR versus $A m p$ for various $A$ values (rate $=1.0$, GIR $=$ $\left.0.1, \sigma^{2}=0.05\right)$.

no closed-form estimate of an optimal Amp based on (16). A proper Amp that provides the best BER performance for a given LDPC code can be found empirically through simulation.

We have performed simulations to investigate the postclipping SNR (in terms of the variance of clipped noise) versus Amp relationship for various values of $A$, GIR and, $\sigma^{2}$ (total variance of unclipped noise) for uncoded systems. The results are shown in Figures 5, 6, 7.

We see that with Amp chosen judiciously, the postclipping SNR value is increased and reaches a maximum. The gain is greater for more impulsive noise. Overclipping due to using a small Amp value degrades performance. An Amp value that is too large effects no clipping and hence no reduction in noise variance and improvement of post-clipping SNR. The optimal Amp value appears to fall between 2.5 and 4 . Further simulations were carried out for coded systems with code rate $=0.42, A=0.1$, and GIR $=0.1$. The preliminary simulation results seem to indicate that the optimal Amp value, resulting in a minimum preclipping SNR $\left(E_{b} / N_{o}\right)$ required to achieve a BER of 0.0001 , is around 3.7 (see Figure 8). In practice, the values of $A$ and GIR can be estimated by the receiver, and the optimal value of Amp chosen accordingly. In a stable environment, these parameters can be predetermined by the receiver location and time.

Further tests have been carried out to compare the measured and theoretical post-FFT variances of clipped-noise, which are very close as shown in Figure 9. Figure 4 also presents the BER versus SNR curves for OFDM with noise clipping. A significant improvement is observed when clipping (with $A m p=3.7$ ) is applied. This confirms that clipping works best for highly impulsive noise. It should be noted that the above results are obtained for a code rate of 0.42 . 


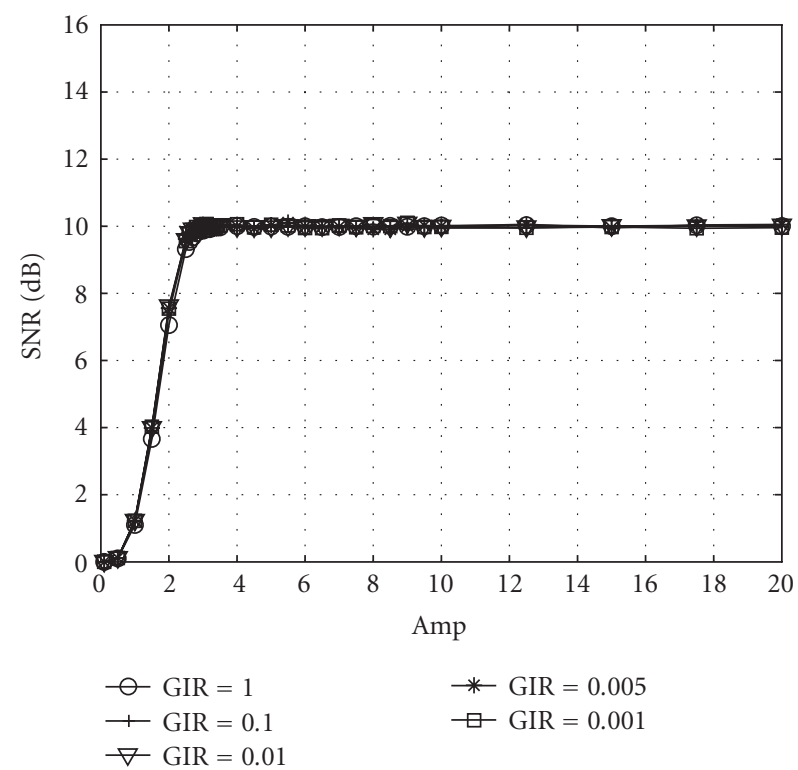

FIgURE 6: SNR versus $A m p$ for various GIR values (rate $=1.0, A=$ $\left.0.1, \sigma^{2}=0.05\right)$.

For a high code rate, say 0.84 , we observe (based on simulations not shown in this paper) that the single carrier system does not perform better than OFDM without clipping due to insufficient redundant parity bits to correct the errors caused by the impulsive noise.

\section{COMPARISON WITH HOMEPLUG 1.0 ROBO MODE}

\subsection{Performance comparison}

In this section, we compare the BER performances between the proposed LDPC-coded system and HomePlug 1.0 ROBO mode. We have carried out computer simulations for both systems using AWCN and a multipath channel with perfect channel-state information (CSI) at the receiver. The proposed LDPC-coded system applies clipping and uses frequency-domain equalization (FEQ) before decoding. The ROBO mode simulation is carried out according to [1]. To make a meaningful comparison with the ROBO mode, we adjust the LDPC code rate such that it equals the rate of the concatenated RS and convolutional code, which is 0.42 with individual rate of 0.84 and 0.5 , respectively. The length of the LDPC code is chosen to be 3312 comparable to the transmission block size used in the ROBO mode. The proposed scheme follows ROBO mode to use 84 evenly spaced carriers in a band from 4.49 to $20.7 \mathrm{MHz}$.

For the impulsive noise, we use the third-order approximation of the AWCN pdf given in (7). The impulsive noise factor $A$ is set to 0.1 and the GIR $=0.1$, both of which have been used in most studies for the highly impaired transmission environment $[4,7]$.

For the multipath channel, we have adopted the four-tap multipath fading model used in [7], assuming the relative

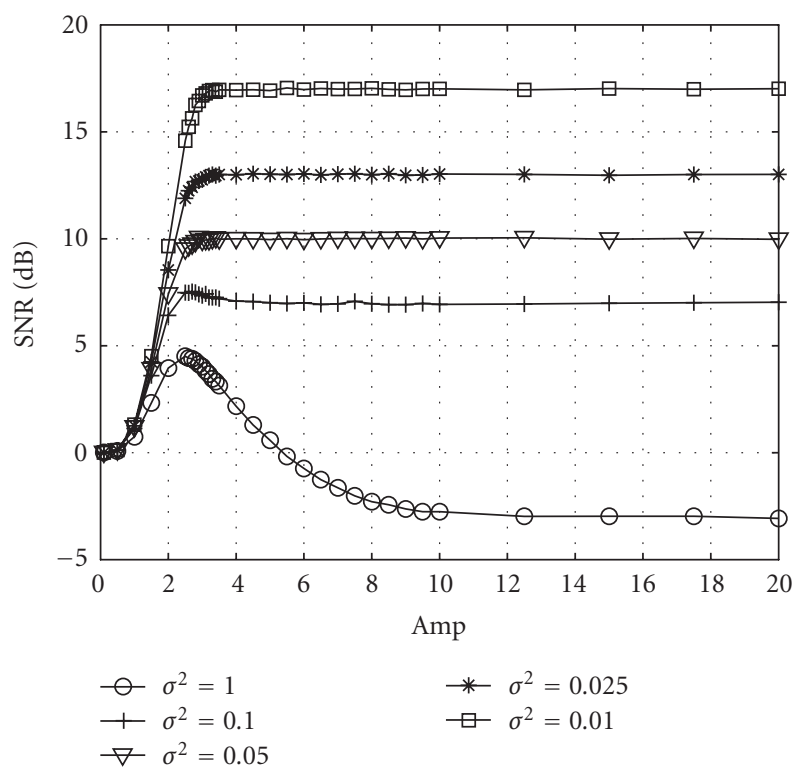

Figure 7: SNR versus $A m p$ for various $\sigma^{2}$ values (rate $=1.0, A=$ $0.1, \mathrm{GIR}=0.1$.

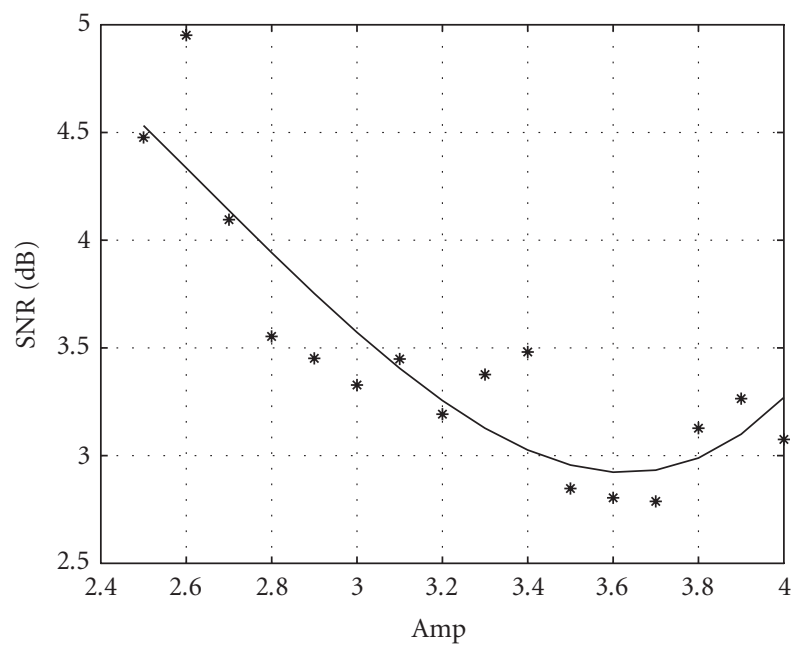

Figure 8: SNR @ BER $=0.0001$ versus $A m p($ rate $=0.42, A=0.1$, GIR $=0.1)$

delay $\tau_{m}$ equal to a multiple of the sample duration $T_{s}$ without loss of generality. The multipath channel output is

$$
\begin{aligned}
y_{n} & =x_{n} * h_{n} \\
& =\sum_{m=0}^{3} \alpha_{m} \exp \left(j \theta_{m}\right) x\left(n-\tau_{m} / T_{s}\right),
\end{aligned}
$$

where $T_{s}=0.05 \mu s, \tau_{m}=0,8 T_{s}, 12 T_{s}, 14 T_{s}, \alpha_{m}=0.2,0.1$, $0.02,0.01$ for $m=0,1,2,3$, and $\theta_{m}$ is uniformly distributed in $[0,2 \pi)$. With this channel, the received signal power is approximately $13 \mathrm{~dB}$ below transmitted signal power. The 


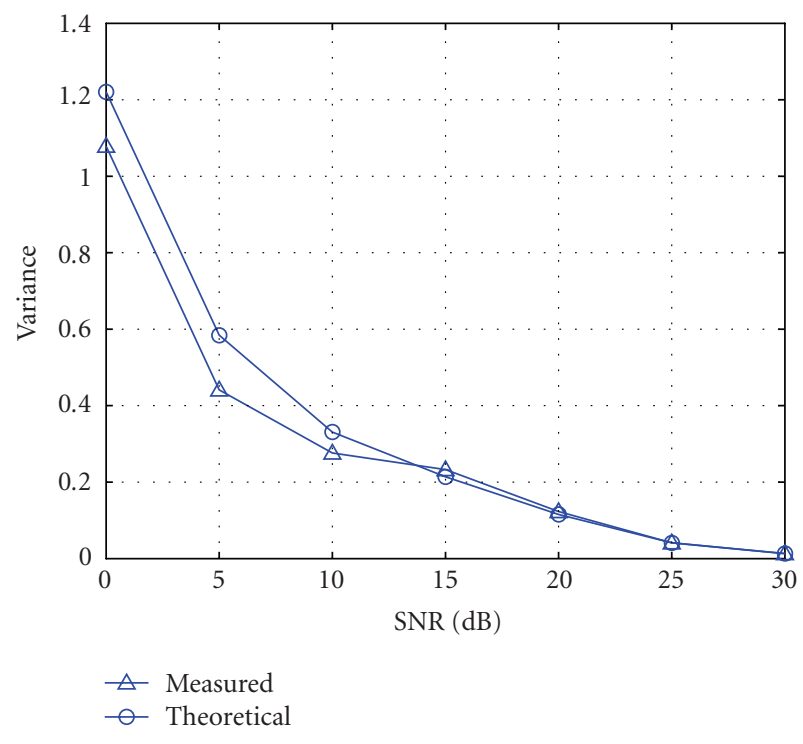

FIGURE 9: Measured and theoretical post-FFT variance of clipped noise over AWCN channel $(A=0.1, \mathrm{GIR}=0.1)$.

channel impulse response is implicitly normalized by measuring SNR at the receiver.

Simulations are carried out to compare the BER performances over an AWCN channel (no multipath) with $A=0.1$ and GIR $=0.1$. It can be seen from Figure 10 that the proposed system achieves a BER of $10^{-6}$ at about $3 \mathrm{~dB}$ SNR, compared with $14 \mathrm{~dB}$ needed by ROBO mode; the proposed system achieves a performance gain of more than $10 \mathrm{~dB}$ at this BER. The performances of the two systems are also compared over the multipath channel with AWGN (no impulsive noise). As shown in Figure 10, with perfect CSI, both systems reach a waterfall performance at SNR between 2$4 \mathrm{~dB}$. However, the LDPC-coded system still achieves a gain of $1 \mathrm{~dB}$ at BER of $10^{-6}$. The gain of the LDPC-coded system over ROBO mode is largely attributed to clipping. When clipping is also applied to ROBO mode, the SNR gain of the LDPC-coded system is $1 \mathrm{~dB}$ at BER $=0.0001$ (not shown in Figure 10). Nevertheless, the LDPC-coded system also provides a 4 -fold throughput gain (see Section 5.D).

As a final comparison, simulations are performed for both systems over a combined multipath and AWCN channel. Figure 11 shows that the proposed LDPC-coded system outperforms the ROBO mode by approximately $10 \mathrm{~dB}$ at a BER of $10^{-6}$ without resorting to interleaving and extensive time and frequency diversity. Figure 11 also includes the BER curves for two shortened improper array codes (IAC) [21] to be described in detail later.

\subsection{Computational complexity}

Decoding complexity is considered in this subsection, as SPA decoding may be computation intensive when many iterations are used. The computational complexity per decoded

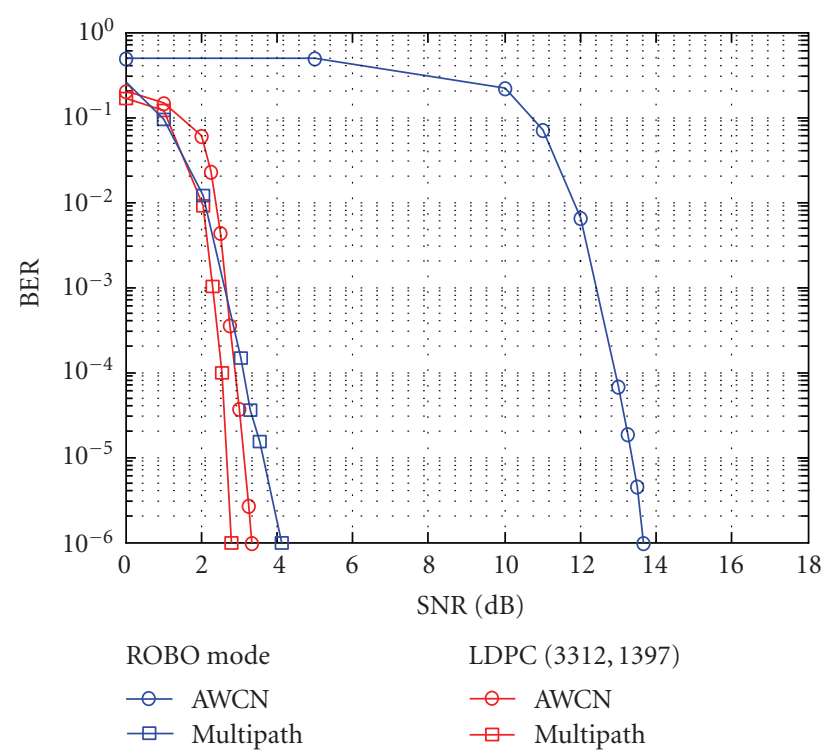

FIgURE 10: BER versus SNR for $\operatorname{LDPC}(3312,1397)$ coded system and ROBO mode over an $\operatorname{AWCN}(A=0.1, \mathrm{GIR}=0.1)$ channel and a multipath channel.



FIGURE 11: BER versus SNR for various LDPC coded systems and ROBO mode over a channel with both AWCN $(A=0.1$, GIR $=0.1)$ and multipath impairments.

bit is defined as the total number of addition, multiplication and division operations. The LDPC code using SPA decoding requires a maximum of 8 iterations to achieve a BER of $10^{-5}$. This amounts to a total of 111 operations per decoded bit. For the ROBO mode, we only count the 
complexity of the trellis-based Viterbi algorithm for decoding the inner convolutional code; doing so flavours the ROBO mode. To achieve the same level of BER of $10^{-5}$ in the ROBO mode, the trellis-based Viterbi decoding for a $(2,1,6)$ convolutional code requires 256 operations per decoded bit for each repeated transmission. Thus, a significant reduction in the computational complexity is obtained using LDPC coding.

\subsection{Implementation issues}

Although SPA allows fast decoding through a parallelprocessing architecture, the MacKay code does not readily lend itself to regular implementation due to its random interconnect patterns between variable and check nodes. It is reported in [22] that the deterministically constructed array code is well suited for parallel implementation of SPA with less memory requirement. The array code is also appealing because of the guaranteed absence of cycles of length four [13]. In [21], shortened array codes are proposed, in which cycles of length $2 k$ can be further eliminated. Two shortened improper array codes are adopted from [21] at code rates of approximately 0.4 and 0.5 , respectively. From Figure 11, it can be seen that at BER of $10^{-6}$ the shortened array code only suffers a performance loss of $0.5-2.5 \mathrm{~dB}$ compared to the MacKay code. Nonetheless, the shortened codes still outperform the ROBO mode by more than $8 \mathrm{~dB}$. The shortened array codes thus serve as a good alternative to the MacKay code.

\subsection{Throughput efficiency}

As described in Section 2.1, the HomePlug 1.0 ROBO mode is usually employed over severely degraded channels. The ROBO mode uses a block interleaver, which is read four times to provide extensive time and frequency diversity and achieve robust operation under hostile conditions. The repetition reduces the data rate to $1 / 4$ bits per carrier per symbol. Consequently, the ROBO mode delivers reliability at the expense of throughput. In the proposed LDPC-coded system, the variable bits are randomly assigned to the check nodes to perform parity-check sum operations, so that interleaving is implicitly incorporated in the random-like structure of the encoding scheme. Not needing repeated transmission, the proposed scheme offers a 4 -fold increase in throughput over ROBO mode.

\section{CONCLUSION}

We have investigated the performance of OFDM with LDPC codes over channels with impulsive noise and multipath fading, as a candidate for improvement over HomePlug 1.0 ROBO mode. First, we establish that noise clipping is advantageous in PLC with severe impulsive noise. A simple and effective scheme is proposed to estimate the variance of the clipped noise for LDPC decoding. We have compared the BER of the proposed LDPC-coded system and the HomePlug 1.0 ROBO mode over an AWCN and multipath channel. The results show that the proposed scheme outperforms HomePlug 1.0 ROBO mode while reducing the decoder computational complexity. In addition, the proposed scheme offers 4 -fold increase in throughput over ROBO mode.

\section{ACKNOWLEDGMENT}

The authors would like to thank the Editor Dr. Lutz Lampe and the reviewers for their valuable comments that helped to improve this paper.

\section{REFERENCES}

[1] M. K. Lee, R. E. Newman, H. A. Latchman, S. Katar, and L. Yonge, "HomePlug 1.0 powerline communication LANs - protocol description and performance results," International Journal of Communication Systems, vol. 16, no. 5, pp. 447-473, 2003.

[2] M. Ardakani, F. R. Kschischang, and W. Yu, "Low-density parity-check coding for impulse noise correction on powerline channels," in Proceedings of the 9th International Symposium on Power Line Communications and Its Applications (ISPLC '05), pp. 90-94, Vancouver, Canada, April 2005.

[3] D. Umehara, H. Yamaguchi, and Y. Morihiro, "Turbo decoding over impulsive noise channel," in Proceedings of the 8th International Symposium on Power Line Communications and Its Applications (ISPLC '04), pp. 51-56, Zaragoza, Spain, MarchApril 2004.

[4] H. Nakagawa, D. Umehara, S. Denno, and Y. Morihiro, "A decoding for low density parity check codes over impulsive noise channels," in Proceedings of the 9th International Symposium on Power Line Communications and Its Applications (ISPLC '05), pp. 85-89, Vancouver, Canada, April 2005.

[5] A. D. Spaulding and D. Middleton, "Optimum reception in an impulsive interference environment-part I: coherent detection," IEEE Transactions on Communications, vol. 25, no. 9, pp. 910-923, 1977.

[6] J. Häring and A. J. Han Vinck, "Performance bounds for optimum and suboptimum reception under Class-A impulsive noise," IEEE Transactions on Communications, vol. 50, no. 7, pp. 1130-1136, 2002.

[7] Y. H. Ma, P. L. So, and E. Gunawan, "Performance analysis of OFDM systems for broadband power line communications under impulsive noise and multipath effects," IEEE Transactions on Power Delivery, vol. 20, no. 2, part 1, pp. 674-682, 2005.

[8] R. G. Gallager, Low Density Parity Check Codes, MIT Press, Cambridge, Mass, USA, 1963.

[9] S. Lin and D. J. Costello, Error Control Coding, Prentice-Hall, Upper Saddle River, NJ, USA, 2nd edition, 2003.

[10] M. Zimmermann and K. Dostert, "Analysis and modeling of impulsive noise in broad-band powerline communications," IEEE Transactions on Electromagnetic Compatibility, vol. 44, no. 1, pp. 249-258, 2002.

[11] Y. Kou, S. Lin, and M. P. C. Fossorier, "Low-density paritycheck codes based on finite geometries: a rediscovery and new results," IEEE Transactions on Information Theory, vol. 47, no. 7, pp. 2711-2736, 2001.

[12] D. J. C. MacKay, "Good error-correcting codes based on very sparse matrices," IEEE Transactions on Information Theory, vol. 45, no. 2, pp. 399-431, 1999. 
[13] J. L. Fan, "Array codes as low-density parity-check codes," in Proceedings of the 2nd International Symposium on Turbo Codes and Related Topics (ISTC '00), pp. 543-546, Brest, France, September 2000.

[14] E. Eleftheriou and S. Ölçer, "Low-density parity-check codes for digital subscriber lines," in Proceedings of IEEE International Conference on Communications (ICC '02), vol. 3, pp. 1752-1757, New York, NY, USA, April-May 2002.

[15] T. J. Richardson and R. L. Urbanke, "Efficient encoding of lowdensity parity-check codes," IEEE Transactions on Information Theory, vol. 47, no. 2, pp. 638-656, 2001.

[16] D. Umehara, M. Kawai, and Y. Morihiro, "An iterative detection for M-ary SS system over impulsive noise channel," in Proceedings of the 6th International Symposium on Power-Line Communications and Its Applications (ISPLC '02), pp. 203207, Athens, Greece, March 2002.

[17] J. Häring and A. J. Han Vinck, "Iterative decoding of codes over complex numbers for impulsive noise channels," IEEE Transactions on Information Theory, vol. 49, no. 5, pp. 12511260, 2003.

[18] H. Suraweera, C. Chai, J. Shentu, and J. Armstrong, "Analysis of impulsive noise mitigation techniques for digital television systems," Research Paper, Department of Electronic Engineering, La Trobe Univeristy, Melbourne, Australia, 2003.

[19] A. Johnson and N. Thomopoulos, "Characteristics and tables of the doubly-truncated normal distribution," Faculty Working Paper, Stuart Graduate School of Business, Illinois Institute of Technology, Chicago, Ill, USA, 2001.

[20] D. Dardari, V. Tralli, and A. Vaccari, "A theoretical characterization of nonlinear distortion effects in OFDM systems," IEEE Transactions on Communications, vol. 48, no. 10, pp. 17551764, 2000.

[21] O. Milenkovic, N. Kashyap, and D. Leyba, "Shortened array codes of large girth," IEEE Transactions on Information Theory, vol. 52, no. 8, pp. 3707-3722, 2006.

[22] S. Ölçer, "Decoder architecture for array-code-based LDPC codes," in Proceedings of IEEE Global Telecommunications Conference (GLOBECOM '03), vol. 4, pp. 2046-2050, San Francisco, Calif, USA, December 2003.

Christine Hsu was born in Ottawa, Canada, in 1980. She graduated with a B.S. degree in Applied Sciences (Electrical Engineering) from the University of Ottawa in May 2003. She received her M.S. degree (Engineering) from the Department of Electrical and Computer Engineering, Queen's University, Kingston, Ontario, Canada, in May 2006. Since June 2006, she has been a Spectrum Engineer with the Canada Department of Industry, Ottawa, Canada.

Neng Wang received the B.Eng. degree from Shanghai Jiao Tong University, Shanghai, China, in 1994, the M.Eng. degree from Nanjing University of Posts and Telecommunications (NUPT), Nanjing, China, in 1999, and the Ph.D. degree from Queen's University, Kingston, Ontario, Canada in 2005, all in Electrical Engineering. He was an Electronics Engineer in Suzhou Lambeau High-Tech Enterprise Group, Suzhou,

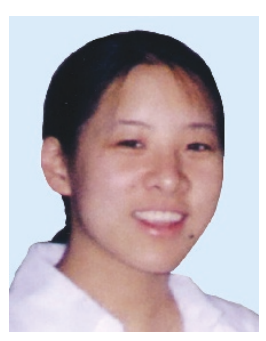
China, from 1994 to 1996 . He has been Research and Teaching

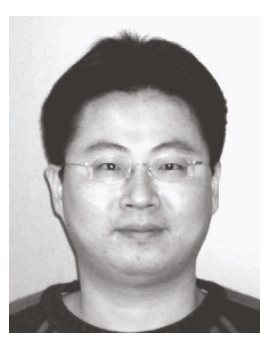

Assistant in the Department of Information Engineering, NUPT from 1996-1999, and the Department of Electrical and Computer Engineering, Queen's University from 2000-2005. He was a Research Scientist at the Communications Research Centre, Ottawa, ON, in 2006. Since 2007, he has been with Nortel Networks, Richardson, TX, as a Wireless Access Standards Engineer. His research interests lie in general areas of wireless communications and signal processing.

Wai-Yip Chan, also known as Geoffrey Chan, received his B.Eng. and M.Eng. degrees from Carleton University, Ottawa, and his Ph.D. degree from University of California, Santa Barbara, all in Electrical Engineering. He is currently with the Department of Electrical and Computer Engineering, Queens University. He has held positions in academia and industry, namely McGill University, Illinois Institute of Tech-

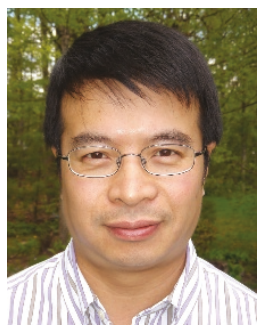
nology, Bell Northern Research, and Communications Research Centre. His research interests are in multimedia signal coding and communications. He is an associate editor of EURASIP Journal on Audio, Speech, and Music Processing. He has helped to organize IEEE sponsored conferences in speech coding, image processing, and communications. He held a CAREER Award from the National Science Foundation.

Praveen Jain received the B.E. degree with honors from the University of Allahabad, India, the M.A.S. and Ph.D. degrees from the University of Toronto, Canada, in 1980, 1984, and 1987, respectively, all in electrical engineering. Presently, he is a Professor and Canada Research Chair in Power Electronics at Queen's University in Kingston, Ontario, Canada. From 1994 to 2000, he was a Professor at Concordia University, Mon-


treal, Canada. Prior to this (1989-1994) he was a Technical Advisor with the Power Group, Nortel Networks, Ottawa, Canada, where he was providing guidance for research and development of advanced power technologies for telecommunications. During 1987-1989, he was with Canadian Astronautics Ltd., Ottawa, Canada, where he played a key role in the design and development of high frequency power conversion equipments for the Space Station Freedom. Dr. Jain has published over 300 technical papers and has 30 patents. His current research interests are power electronics applications to space, telecommunications and computer systems. He is a member of Professional Engineers of Ontario and an Associate Editor of IEEE Transactions on Power Electronics. He is a Fellow of the Institute of Electrical and Electronics Engineers and the recipient of the 2004 Engineering Medal of Ontario Professional Engineers. 